\title{
MTT and Blood-Brain Barrier Disruption within Asymptomatic Vascular WM Lesions
}

\author{
(D) B.E. Dewey, (D)X. Xu, (D) K. Knutsson, (D) A. Jog, (D).L. Prince, (DP.B. Barker, (DP.C.M. van Zijl, (D) R. Leigh, and (DP. Nyquist
}

\begin{abstract}
BACKGROUND AND PURPOSE: White matter lesions of presumed ischemic origin are associated with progressive cognitive impairment and impaired BBB function. Studying the longitudinal effects of white matter lesion biomarkers that measure changes in perfusion and BBB patency within white matter lesions is required for long-term studies of lesion progression. We studied perfusion and BBB disruption within white matter lesions in asymptomatic subjects.
\end{abstract}

MATERIALS AND METHODS: Anatomic imaging was followed by consecutive dynamic contrast-enhanced and DSC imaging. White matter lesions in 21 asymptomatic individuals were determined using a Subject-Specific Sparse Dictionary Learning algorithm with manual correction. Perfusion-related parameters including CBF, MTT, the BBB leakage parameter, and volume transfer constant were determined.

RESULTS: MTT was significantly prolonged (7.88 [SD, 1.03] seconds) within white matter lesions compared with normal-appearing white (7.29 [SD, 1.14] seconds) and gray matter (6.67 [SD, 1.35] seconds). The volume transfer constant, measured by dynamic contrast-enhanced imaging, was significantly elevated $\left(0.013[\mathrm{SD}, 0.017]\right.$ minutes $\left.^{-1}\right)$ in white matter lesions compared with normalappearing white matter $\left(0.007\right.$ [SD, 0.011] minutes $\left.^{-1}\right)$. BBB disruption within white matter lesions was detected relative to normal white and gray matter using the DSC-BBB leakage parameter method so that increasing BBB disruption correlated with increasing white matter lesion volume (Spearman correlation coefficient $=0.44 ; P<.046$ ).

CONCLUSIONS: A dual-contrast-injection MR imaging protocol combined with a 3D automated segmentation analysis pipeline was used to assess BBB disruption in white matter lesions on the basis of quantitative perfusion measures including the volume transfer constant (dynamic contrast-enhanced imaging), the BBB leakage parameter (DSC), and MTT (DSC). This protocol was able to detect early pathologic changes in otherwise healthy individuals.

ABBREVIATIONS: $\mathrm{CSVD}=$ cerebrovascular small-vessel disease; $\mathrm{DCE}=$ dynamic contrast-enhanced; $\mathrm{Gd}=$ gadolinium; $\mathrm{K}_{2}=$ BBB leakage parameter; $K^{\text {trans }}=$ volume transfer constant; WML $=$ white matter lesion

U

nderstanding vascular contributions that influence cognitive decline and dementia is a national research priority. ${ }^{1,2}$ Cerebrovascular small-vessel disease (cSVD) is associated with stroke and dementia and is potentially modifiable. ${ }^{3-5}$ Many aspects of vascular disease of the brain can be detected with MR

Received October 16, 2020; accepted after revision March 13, 2021.

From the Departments of Electrical and Computer Engineering (B.E.D., J.L.P.) and Radiology and Radiological Science (X.X., L.K., J.L.P., P.B.B., P.C.M.v.Z.), Division of MRI Research, and Department of Neurology (R.L., P.N.), Johns Hopkins University, Baltimore, Maryland; F.M. Kirby Research Center for Functional Brain Imaging (B.E.D., X.X., P.B.B., P.C.M.v.Z.), Kennedy Krieger Institute, Baltimore, Maryland; Department of Medical Radiation Physics (L.K.), Lund University, Lund, Sweden; and Athinoula A. Martinos Center for Biomedical Imaging (A.J.), Harvard University Medical School, Boston Massachusetts.

B.E. Dewey and X. Xu contributed equally to this work.

This work was supported by funding from the National Institutes of HealthNational Institute of Neurological Disorders and Stroke (R01NS062059) and the Johns Hopkins Department of Anesthesia, Stimulating and Advancing ACCM Research award. imaging. Features associated with cSVD include small subcortical (lacunar) infarcts, white matter hyperintensities, dilated perivascular spaces, microbleeds, brain atrophy, and increased BBB permeability. ${ }^{6,7}$ White matter lesions (WMLs) seen on T2weighted MR imaging are the most common feature of cSVD, estimated to represent $40 \%$ of cSVD disease burden. ${ }^{6}$ WMLs are accompanied by many pathologic changes, including $\mathrm{BBB}$ disruption. $^{8-17}$ While other multifactorial pathophysiologic mechanisms are undoubtedly involved, including hypertension, genetic factors, and inflammation, ${ }^{18-25}$ changes in $\mathrm{CBF}$ and

Please address correspondence to Paul Nyquist, MD, Johns Hopkins, Department of Neurology, 416 Phipps, 600 N Wolfe St, Baltimore MD 21287; e-mail: pnyquis1@jhmi.edu

\footnotetext{
- Indicates open access to non-subscribers at www.ajnr.org

Indicates article with online supplemental data.

http://dx.doi.org/10.3174/ajnr.A7165
} 
increasing BBB permeability have been implicated as markers of WML progression and may have a causative role. ${ }^{13,26}$

Quantifying different measures of hemodynamics such as CBF, $\mathrm{CBV}$, and BBB disruption directly within WMLs has been difficult. Previous studies have shown decreased CBF in larger brain regions associated with WMLs but not within WMLs themselves. ${ }^{6,7,17,26-32}$ These studies have also identified increased regional nonlesional volume transfer constant ( $K^{\text {trans }}$ ) using gadolinium $(\mathrm{Gd})$-based dynamic contrast-enhanced (DCE) MR imaging, but these methods have drawbacks such as decreased signal discrimination within and without WMLs and a dependence on adequate correction for decreased perfusion within WMLs. ${ }^{7,31,33}$ Some studies have detected decreased CBF within lesions using arterial spinlabeling, ${ }^{34}$ while others have detected these changes within regions surrounding WMLs and within ROIs within WMLs. ${ }^{26,35}$

Presently, new imaging approaches and data-processing pipelines are needed to allow us to segment WMLs and measure subtle intralesion changes in $\mathrm{CBF}, \mathrm{MTT}$, and $\mathrm{BBB}$ disruption. Measures of $\mathrm{BBB}$ permeability incorporate MR imaging surrogates, which detect $\mathrm{Gd}$ extravasation outside the microvasculature due to disruption of the BBB related to microvascular injury. ${ }^{7,31}$ In the current study, on a voxel-by-voxel basis, we quantify 2 different parameters related to tissue abnormality: $K^{\text {trans }}$ from DCE MR imaging and the BBB leakage parameter $\left(\mathrm{K}_{2}\right)$ from DSC MR imaging, ${ }^{28,29}$ which can relate changes in $\mathrm{BBB}$ transport and/or $\mathrm{CBF}^{36}$ These values can then be assessed for WMLs to get insight into changes in BBB functioning and tissue perfusion. In addition, we assessed MTT, which reflects tissue perfusion.

In the Genetic Study of Atherosclerosis Risk (GeneSTAR) cohort study, we have identified individuals with a family history of early-onset coronary vascular disease with earlier WMLs detected in midlife, ${ }^{37}$ with a concomitant impact on measures of cognitive-motor function. ${ }^{38}$ In this relatively young high-risk subgroup (average age, 54.1 [SD, 3.5] years) of 21 participants with repeat MR imaging, we have observed rapid rates of WML progression associated with cognitive decline. ${ }^{39}$ In this study, we present a data-analysis pipeline that incorporates segmentation of WMLs $^{40,41}$ and quantification of perfusion-based measures of MTT, CBF, $\mathrm{K}_{2}$, and $K^{\text {trans }}$ from both DSC and DCE MR imaging. This work builds on previous work measuring microvascular perfusion and Gd extravasation in different regions of the brain. ${ }^{31,42}$ We propose that these Gd-based representations of BBB disruption in WMLs, with knowledge of the CBF, may enable identifying WMLs at risk of progression at a stage at which they may respond to strategies of disease prevention. ${ }^{3,4,6}$

\section{MATERIALS AND METHODS \\ Patient Recruitment}

The 21 participants in this repeat imaging study were healthy family members of relatives with known early-onset coronary artery disease who were randomly selected (2008-2013) from a previous GeneSTAR WML study and recruited. ${ }^{43}$ The study was approved by the institutional review board and was conducted at the Johns Hopkins medical campus in Baltimore, Maryland. ${ }^{44}$ The parent study was designed to characterize the genetic and biologic traits associated with incident cardiovascular disease. ${ }^{38}$ In the larger GeneSTAR study, probands were identified during hospitalization for an acute coronary syndrome or acute myocardial infarction or with angiographic evidence of a flow-limiting stenosis before 60 years of age. Probands did not participate in the final study by design. Apparently healthy asymptomatic siblings, their offspring, and the offspring of the probands were eligible if they were 29-75 years of age and had no personal history of coronary artery disease, stroke, or TIAs. The 21 participants in this study were recruited from a cohort of 714 participants in a previous MR imaging substudy from the larger GeneSTAR study population (54.1 [SD, 3.5] years, $45 \%$ hypertensive). Written consent was obtained from each participant.

\section{MR Imaging}

The participants were examined using a 3T Achieva MR imaging scanner (Philips Healthcare) with a 32-channel head coil. The examination protocol is illustrated in the Online Supplemental Data. The structural series included axial T1-weighted MPRAGE and axial turbo spin-echo FLAIR as follows: The MPRAGE sequence was chosen over other T1WI sequences due to its excellent GM/ WM contrast. The sequence parameters are the following: 1) axial T1-weighted MPRAGE: flip angle $=8^{\circ}, \mathrm{TR}=10 \mathrm{~ms}, \mathrm{TE}=6 \mathrm{~ms}$, voxel size $=1.0 \times 1.0 \times 1.0 \mathrm{~mm}$, contiguous slices, $\mathrm{FOV}=$ $240 \times 240 \times 160 \mathrm{~mm}$, and reconstruction matrix $=320 \times$ $320 \times 160$; and 2) axial turbo spin-echo FLAIR: TR $=11,000 \mathrm{~ms}$, $\mathrm{TI}=2800 \mathrm{~ms}, \mathrm{TE}=68 \mathrm{~ms}$, voxel size $=0.98 \times 0.98 \times 3.0 \mathrm{~mm}$, contiguous slices, $\mathrm{FOV}=240 \times 240 \times 132 \mathrm{~mm}$, and reconstruction matrix $=512 \times 512$. The Online Supplemental Data show an example of the MPRAGE, FLAIR, precontrast baseline DCE, and DSC images from 1 participant. The structural images were reviewed by the Principal Investigator to ascertain any health concerns. The examinations were follow-up MRIs obtained after a prior MR imaging that had been reviewed by a neuroradiologist and were deemed to have normal findings with the exception of signs of WM hyperintensity and age-related microvascular disease. No lacunar infarcts were detected in this study cohort by clinical radiologists and the study investigator.

T1 mapping was performed before contrast agent injection with an inversion recovery Look-Locker sequence at resolution of $2 \times 2.2 \times 4 \mathrm{~mm}^{3}$ with a flip angle of $4^{\circ}$ and a TR of 2.6 seconds. The first TI was $38.5 \mathrm{~ms}$, and the spacing between successive time points was $69 \mathrm{~ms}$. Thirty-eight images with different TIs were recorded for a total of 11 slices. The total scan time was 112 seconds.

A T1-weighted gradient-echo sequence was used for DCE imaging using the following parameters: flip angle $=26^{\circ}, \mathrm{TE}=2.5 \mathrm{~ms}$, $\mathrm{TR}=5.1 \mathrm{~ms}$, resolution $=22 \times 2.22 \times 4 \mathrm{~mm}^{3}$. Gadoteridol (ProHance; Bracco Diagnostics) was given at a dosage of $0.1 \mathrm{mmol} /$ $\mathrm{kg}$ via a power injector. The contrast agent was injected with an injection delay of 30 seconds (14 precontrast baseline images) at a speed of $5 \mathrm{~mL} / \mathrm{s}$, followed by a $20-\mathrm{mL}$ saline rinse with the same speed. The time for acquiring each dynamic scan was 2.5 seconds for 11 slices, and a total of 150 dynamic series were acquired. A postcontrast MPRAGE image was acquired after the Gd contrast injection.

Approximately 6 minutes after the DCE scan, DSC imaging was performed using single-shot EPI with $\mathrm{TE}=29 \mathrm{~ms}$, TR $=$ $1500 \mathrm{~ms}$, flip angle $=90^{\circ}$, and resolution $=2 \times 2.2 \times 4 \mathrm{~mm}^{3} \mathrm{~A}$ 
second dose of gadoteridol was injected with a 15-second injection delay (10 precontrast baseline images) at a speed of $5 \mathrm{~mL} / \mathrm{s}$, followed by a $20-\mathrm{mL}$ saline rinse with the same speed. The time for acquiring each dynamic scan was 1.5 seconds, and a total of 80 dynamic series were acquired for 25 slices covering most of the brain. As a direct comparison with the precontrast image, the MPRAGE sequences were repeat postcontrast, with the same parameters as those in precontrast, for direct identification of any lesion enhancement.

\section{Data Processing}

Anatomic volumes of discrete brain regions and tissue types were determined with MPRAGE images, and WML volumes were determined using FLAIR images coregistered into Montreal Neurological Institute space. ${ }^{45}$ Spatial normalization of coregistered MPRAGE and FLAIR images into Montreal Neurological Institute space was performed via affine transformation. MPRAGE images were skull-stripped and coregistered to FLAIR images. Within this pilot study, there was some enlargement of perivascular spaces observed, but they were not systematically quantified.

\section{DCE and DSC Postprocessing}

DSC, DCE dynamic images, and T1 maps were processed using nordicICE (NordicNeuroLab). For each participant, first, a precontrast T1 map was calculated by fitting the Look-Locker inversion recovery images to a model. The DCE dynamic images were motion-corrected, and the $K^{\text {trans }}$ maps were calculated using the extended Tofts model. ${ }^{46}$ The arterial input function used in the extended Tofts model was defined by taking the average of semiautomatically selected voxels ( 2 or 3 voxels) within the anterior cerebral arteries for each participant in the DCE images.

To calculate the CBF and MTT maps, we processed the DSC images for perfusion parameters using delay-insensitive singular value deconvolution with a threshold of 0.15 for regularization. ${ }^{47}$ The arterial input function was defined by taking the average of semi-automatically selected voxels (5 voxels) within the branches of the middle cerebral artery around the Sylvian fissure. The reason for not choosing the same arteries as in DCE is that DSC is subjected to distortions due to the EPI readout in the frontal part of the brain due to susceptibility artifacts. No leakage correction was applied for the postprocessing.

The pre-infusion images from the DCE and DSC scans were averaged and coregistered to the MPRAGE image using a fully automated pipeline, BrainMap (http://brainmap.org/software.html). ${ }^{48}$ The pipeline performed image registration using the Advanced Normalization Tools (ANTs; http://stnava.github.io/ANTs) software package, ${ }^{49}$ skull removal using Multi-cONtrast brain STRipping (MONSTR; https://www.nitrc.org/projects/monstr/), ${ }^{50}$ whole-brain gray/white matter segmentation using Multi-Atlas Cortical Reconstruction Using Implicit Surface Evolution (MaCRUISE; https://github.com/MASILab/MaCRUISE), ${ }^{51}$ and lesion segmentation using the Subject-Specific Sparse Dictionary Learning (S3DL; http://iacl.ece.jhu.edu/index.php?title=Subject_Specific_ Sparse_Dictionary_Learning_for_Atlas_Based_Brain_MRI_ Segmentation $){ }^{41}$ Automatically segmented lesion masks were manually edited to remove false-positives common to this process. Segmentation masks were eroded (except for lesion masks) and applied to parameter maps to extract ROIs representing normal-appearing WM, GM, and WMLs. To extract parameter values for each participant, we took the median voxel value in each ROI from the CBF, MTT, and $K^{\text {trans }}$ maps.

In addition to calculating perfusion metrics, DSC images were also separately processed to calculate $\mathrm{K}_{2}$ values. ${ }^{52}$ DSC images are $\mathrm{T} 2^{\star}$-weighted, and intravascular gadolinium causes a decrease in signal due to its susceptibility artifacts. However, DSC images also have some T1-weighting that is proportional to the concentration of gadolinium in the tissue. ${ }^{32}$ During a DSC acquisition, the measured change in signal is due to both intravascular gadolinium (flow-dependent) and parenchymal gadolinium (leakdependent). ${ }^{53}$ Using normal tissue as a reference, one can isolate the signal due to gadolinium leakage from the signal due to intravascular gadolinium. ${ }^{52}$ Normal tissue can be identified by excluding voxels that exhibit signal changes due to gadolinium leakage. ${ }^{28}$ In our case, in which leakage is small and limited to a few white matter regions, a "normal brain DSC response curve" was defined from a whole-volume analysis (about 3000 voxels). Subsequently, arrival time correction ${ }^{52}$ was applied to each individual voxel by scaling (width and height of the initial response) and shifting (position of the peak maximum of the initial response) the DSC dynamic curves. $\mathrm{K}_{2}$ in each voxel was then determined using

$$
\Delta \widetilde{R} 2^{*}(t)_{A T C}=\overline{\Delta R 2^{*}}(t)-K_{2} \int_{0}^{t} \overline{\Delta R 2^{*}}\left(t^{\prime}\right) d t^{\prime},
$$

in which $\Delta \widetilde{R} 2^{*}(t)_{A T C}$ is the corrected change in relaxivity and $\overline{\Delta R 2^{*}}(t)$ is the average signal of the normal brain. Thus, $\mathrm{K}_{2}$ reflects the proportion of the recorded signal that is due to gadolinium leakage. The resulting voxel-by-voxel measure of $\mathrm{K}_{2}$ was used to generate a blood-brain permeability image in which voxels were assigned as normal when the fitted $\mathrm{K}_{2}$ was $<0.1 \%$.

FLAIR images were coregistered to the DSC source images using a diffeomorphic registration pipeline. ${ }^{54}$ By means of the combined transforms, the WML ROIs were moved from FLAIR space to DSC space. The mean $\mathrm{K}_{2}$ value for all voxels within the WML ROIs that demonstrated elevated BBB permeability (gadolinium leakage) was calculated for each patient and used in the subsequent analysis. The $\mathrm{K}_{2}$ analysis was performed on the DSC acquisition that occurred during the second dose of gadolinium administration. $\mathrm{K}_{2}$ is largely a first-pass measure as opposed to $K^{\text {trans }}$, which is measured in steady-state. Thus, for this study, the measured $\mathrm{K}_{2}$ reflects gadolinium leakage that occurred during the second injection and beyond steady-state background from the first injection.

\section{Statistical Analysis}

All statistical analyses were performed using $\mathrm{R}$ statistical and computing software (http://www.r-project.org/). ${ }^{55}$ Linear mixed effects modeling was used for statistical comparison among WM, GM, and WMLs based on the ROI level. Significance levels were assessed using paired Wilcoxon signed rank tests. Spearman correlations coefficients were calculated between lesion volumes and subjects. 


\section{RESULTS}

All scans were read by the investigator and then read formally by a clinical neuroradiologist. There were no lacunar infarcts detected in this small study. Within this small group included in the pilot study, there was a significant quantity of atrophy observed and there was some enlargement of perivascular spaces observed, but these were not systematically quantified.

T1 MPRAGE images were used for GM and WM segmentation, and FLAIR images were used for lesion assignment. Notice that some lesions are not highlighted due to the size of the lesions and the detection limitations of the algorithm. The nonsegmented lesions were not included in the analysis. None of the subjects' images showed any contrast enhancement on the postcontrast MPRAGE. Two subjects were excluded due to technical problems in the contrast agent administration. Representative images for the CBF, MTT, and $K^{\text {trans }}$ maps, and their overlays on anatomic images of 1 participant are presented in the Online Supplemental Data. The perfusion images were interpolated to match the FLAIR resolution. MTT values are elevated within and around the clusters of WMLs (Online Supplemental Data). While all ROIs followed an approximately normal distribution, given the limited sample size, the median values were calculated instead of the mean. The boxplot in the Online Supplemental Data shows the median and interquartile range of MTT in the GM, WM, and WMLs of all participants. Paired Wilcoxon signed rank tests reveal that the MTT is significantly prolonged $(P<.001)$ in WMLs (7.88 [SD, \pm 1.03$]$ seconds, median + interquartile range) compared with normal-appearing WM (6.67 [SD, 1.35] seconds), suggesting that these areas of the brain have associated vascular pathology. We also see that there is a small-but-significant difference $(P<.001)$ in MTT for the reference WM and GM (7.29 [SD, 1.14] seconds) ROIs. CBF values were also calculated, and we found that there is no significant difference in CBF between WMLs and WM $(P=.62)$.

The Online Supplemental Data show boxplots of the mean values for each ROI for $K^{\text {trans }}$ for all participants. $K^{\text {trans }}$ showed a much larger variance between subjects with smaller difference between ROIs. The $K^{\text {trans }}$ values were significantly different $(P<.001)$ between the WMLs $\left(0.013\right.$ [SD, 0.017] minutes $\left.^{-1}\right)$ and the normal-appearing WM (0.007 [SD, 0.011] minutes $\left.^{-1}\right)$.

The Online Supplemental Data show an example of colorcoded $\mathrm{K}_{2}$ values overlaid on a gray-scale DSC source image. Voxels in the WMLs are shown in color, with increasing BBB disruption going from green (least severe) to yellow to orange to red (most severe). The Online Supplemental Data show the signal change $(\Delta \mathrm{R})$ with time (at each dynamic) of the recorded signal (red dashed lines) and the normal average signal (solid blue lines) before and after applying the arrival time correction. The Online Supplemental Data are for a voxel with a $\mathrm{K}_{2}$ of $0.1 \%$ (a green voxel from panel A). The Online Supplemental Data are for a voxel with a $\mathrm{K}_{2}$ value of $3.5 \%$ (a red voxel in panel A). Note that in the setting of $\mathrm{BBB}$ disruption, the dashed line is pulled down below the baseline due to the $\mathrm{T} 1$ effects from contrast leakage into the tissue. Using the DSC- $\mathrm{K}_{2}$ technique, we averaged voxels within segmented WMLs with elevated $\mathrm{K}_{2}$. The average $\mathrm{K}_{2}$ value across the cohort was 2.67 [SD, 2.33\%]. This value was correlated with 3 variables: total WML volume, subcortical WML volume, and periventricular WML volume. Increasing average $\mathrm{K}_{2}$ was significantly correlated with total WML volume (Spearman correlation $=0.44 ; P<.046$ ), and there was a trend toward correlation with periventricular WML volume (Spearman correlation $=0.44$; $P<.071)$. No significant correlation with subcortical WML volume (Spearman correlation $=0.0078 ; P<.973$ ) was found.

\section{DISCUSSION}

The underlying pathophysiology causing cSVD and WMLs has been attributed to intermittent ischemia due to microvascular narrowing and altered compliance leading to transient hypoperfusion in vulnerable watershed zones. ${ }^{4,26,31}$ Exogenous contrastbased perfusion MR imaging has been used extensively to image hemodynamic changes in the microvasculature of ischemic white matter disease. ${ }^{4,27,31,32,34,35,42,56,57}$ Most studies have taken the strategy of quantifying perfusion parameters in different regions of the whole brain, as opposed to measuring these parameters within WMLs, and comparing them with overall WML burden. ${ }^{14,27,34,35,42}$ While a few studies have separately used arterial spin-labeling ${ }^{34}$ and Gd-based perfusion MR imaging within lesions, ${ }^{4,27,35,42,56}$ these lesion studies differ from ours in that they either used strategies focused on limited ROIs, including nonlesional white matter, or determined the WML volume using manual readers. ${ }^{25,27,31,54,58}$

Our approach is different from the approaches in these other studies in that we used an automated WML segmentation pipeline allowing us to determine the CBF, MTT, $\mathrm{K}_{2}$, and $K^{\text {trans }}$ within individual WMLs and compare them with normal-appearing WM and GM. ${ }^{41,45,59}$ Automated technology is required to study perfusion parameters and BBB disruption in large epidemiology studies to ensure consistency and speed in analysis. ${ }^{4,6,13}$ Our study design allows one to obtain perfusion parameters such as $\mathrm{CBF}, \mathrm{MTT}, \mathrm{K}_{2}$, and $K^{\text {trans }}$ in the same scan session. We found significant increases in MTT and $K^{\text {trans }}$, but no significant change in CBF within WMLs compared with unaffected GM and WM. Gd leakage detected with $\mathrm{K}_{2}$ indicated more severe BBB disruption in subjects with a larger burden of WMLs.

The measurement of absolute CBF using DSC is difficult due to the lack of a direct linear relationship between contrast concentration and the signal change and partial volume effects in the arterial input function. ${ }^{36}$ Therefore, it is common to use relative values, making it difficult to make comparisons among different studies and cohorts. MTT reflects the average time for the blood to pass through a given region of brain tissue, and it is calculated by dividing the CBV by the CBF or using the Zierler area-toheight relationship. ${ }^{60,61}$ The MTT is measured in seconds, and the reverse MTT reflects the local cerebral perfusion pressure. ${ }^{30}$ MTT removes the need for obtaining absolute values of CBF and $\mathrm{CBV}$; therefore, MTT has the potential to serve as a marker of hemodynamic change in white matter diseases. Tissue with decreased cellularity or metabolic activity could have decreased CBF; for example, Promjunyakul et $\mathrm{al}^{62}$ reported reduced CBF in WMLs as well as the adjacent normal-appearing WM regions beyond the WMLs, using the arterial spin-labeling technique in elderly volunteers (mean age, 84.1 years).

Our study has some differences compared with this article: First, the CBF in our article was measured using DSC MR 
imaging. DSC MR imaging has better contrast-to-noise in white matter than arterial spin-labeling, in which the lower SNR makes the detection of changes less sensitive. ${ }^{63}$ Also, the resolution in the above article is $3 \times 3 \times 4 \mathrm{~mm}^{3}$ compared with $2 \times 2.2 \times 4$ $\mathrm{mm}^{3}$ in our study. Therefore, we could better place the voxels without the risk of partial volume effects. There should not have been a significant contribution of a perilesional zone, if any. Second, because we did not find decreased CBF within WMLs in the current study, it is not likely that the perilesional zone would have decreased the CBF. One significant difference between this article and most other literature is that the patient cohort is relatively young and asymptomatic. We found no change in $\mathrm{CBF}$ between WMLs and normal-appearing WM, while the MTT (CBV/CBF) was elevated, indicating compensatory vasodilation and reduced cerebrovascular resistance due to autoregulation. The elevated MTT observed in the WMLs before CBF changes may be an early indicator of asymptomatic cSVD.

Gadoteridol is a macrocyclic gadolinium-based contrast agent that is approved for multidose application. It was recommended that one-fourth-dose to 1 full-dose contrast agent preload be given 5-10 minutes before the DSC scan to reduce the T1 effect caused by contrast agent extravasation. ${ }^{64}$ In our study, one full dose of gadoteridol was injected, which served as a preload for DSC and also allowed us to perform the DCE experiment. We studied $K^{\text {trans }}$ derived from the DCE experiment using the extended Tofts model to provide more information about BBB disruption. $K^{\text {trans }}$ is a combination parameter that includes both permeability and perfusion. In a perfusion-limiting situation, the measured $K^{\text {trans }}$ may reflect perfusion instead of permeability. It has been shown that during a low-leakage situation, there is a risk that $K^{\text {trans }}$ will be overestimated using the extended Tofts model. ${ }^{65}$ Because we did not observe any visible enhancement, we believe that the risk of overestimation was avoided. We have also found that the CBF was maintained in the WMLs, and the elevated $K^{\text {trans }}$ can be attributed to increased permeability in the WMLs compared with the normal-appearing WM. However, the T1-based DCE experiment is inherently noisier, and the calculation of $K^{\text {trans }}$ requires fitting that may not be robust. In addition, $K^{\text {trans }}$ itself cannot be used to reflect permeability without knowledge of CBF.

In our study, we performed 2 gadolinium injections. DSC images are used to measure perfusion parameters. The signal change on the $\mathrm{R} 2{ }^{*}$-weighted DSC source images during the passage of a gadolinium bolus is dominated by susceptibility effects from the intravascular contrast agent. However, in the presence of gadolinium leakage, the recorded signal is augmented by a T1 effect, acting in the opposite direction, which is proportional to the concentration of gadolinium leakage (Online Supplemental Data). Thus, after arrival time correction, the effect of gadolinium leakage can be isolated as $\mathrm{K}_{2}$ (Online Supplemental Data). $\mathrm{K}_{2}$ reflects the signal change due to gadolinium leakage through the $\mathrm{BBB}$ during the first (and, to a lesser extent, the second) pass of the gadolinium bolus through the brain. Using the DSC- $\mathrm{K}_{2}$ approach has the advantage that the effects of blood flow and BBB permeability are being collected simultaneously during a first pass as opposing signals. In contrast, the DCE- $K^{\text {trans }}$ approach requires interpreting a separately acquired perfusion metric with a steady-state model of T1 signal change in which the effect may not be large enough to be measured in the setting of reduced blood flow. In addition, when the second gadolinium injection was given, the first injection was considered to be at steady-state. Therefore, any leakage detected by $\mathrm{K}_{2}$ for the second injection reflects first-pass effects beyond the background steadystate signal. Using the DSC- $\mathrm{K}_{2}$ method, we found that higher $\mathrm{K}_{2}$ values were significantly correlated with total WML volume, suggesting that in these asymptomatic patients, increasing WML volume is associated with increasing disruption of the BBB.

A recent article by Wong et $\mathrm{al}^{26}$ demonstrated that increased extravasation of Gd correlated with declines in CBF in the perilesional zone of WMLs. This approach solidifies the association of hypoperfusion with lesion progression and BBB disruption. The Patlak graphic method used in the article to measure Gd transition into the lesion is perfusion-dependent. Even though $K_{2}$ is a dimensionless, relative measure, it has the advantage that $K_{2}$ is not dependent on perfusion and is suited for detection of smaller $\mathrm{BBB}$ breakdown effects in regions of lower CBF. In our study, manual detection of increasing $\mathrm{K}_{2}$ was observed in proximity to WML borders as well but was not quantifiable in an automated fashion and could not be associated with declining perfusion in proximity to the WML border. Imaging techniques to simultaneously assess MTT, CBF, CBV, $K^{\text {trans }}$, and $\mathrm{K}_{2}$ are essential to study how changes in perfusion affect WML progression ${ }^{14,27,31,34}$ and allow investigation of how subsequent $\mathrm{BBB}$ breakdown with associated increases in measured $\mathrm{BBB}$ disruptions may precede brain parenchymal injury in WMLs. ${ }^{4,31,42}$ It has been previously observed in other studies that BBB disruption in gray matter and normal-appearing white matter is increased with increasing WML burden and other signs of small-vessel disease and this has been studied extensively in white matter hyperintensities and Alzheimer disease.

Our study was not powered or designed to detect changes BBB disruption/permeability in normal-appearing white matter in general but only to compare existing WMLs and normalappearing white matter BBB disruption using our combined imaging approach. Finding a control group without WMLs within the original study sample is very difficult because $90 \%$ of all participants in this study have signs of white matter hyperintensities, regardless of risk factors. The patients were selected across the 800 or so included in the study. They were selected for the following reasons: high or low white matter hyperintensity burden, age, sex, the presence of hypertension, and ethnicity. They were balanced to reflect the age, race, sex, and relative demographic composition of the original sample. Currently, it is difficult to know how BBB disruption could be used to predict WML progression without a study involving repeat imaging of the participant at 2 separate points in time. In future prospective studies with a larger sample size, one may have the statistical power to establish a permeability threshold above which the WMLs are at risk of expansion or the WM is at risk of lesion formation.

There are several limitations to our study. Our technique incorporated coregistration of segmented lesions of brain WMLs with brain perfusion images. The large section thickness of perfusion scans relative to the higher-resolution FLAIR images may 
have resulted in a partial volume effect. Additionally, some of the smaller lesions were too small to allow accurate measurement of the perfusion characteristics and, thus, were not picked up by the automatic analysis. Due to the small sizes of the lesions and the difficulty in obtaining adequate segmentation in such small areas, lesion masks were not eroded and may include some partial volume on the edges of lesions. Finally, the $K_{2}$ values depend on a time integration of the difference signal for the $\Delta \mathrm{R} 2^{*}$ curves. A disadvantage of this approach is that $K_{2}$ depends on the MR imaging parameters and number of dynamics (length of the curve) used in the analysis. Thus, while the $\mathrm{K}_{2}$ values will provide an accurate reflection of BBB breakdown when used consistently within a study, they can differ in absolute value among studies.

\section{CONCLUSIONS}

The results show that MTT was increased significantly within WMLs compared with normal-appearing GM and WM, while no significant $\mathrm{CBF}$ alteration was found between WMLs and normal-appearing WM. These findings indicate vasodilation within the WMLs to maintain normal blood flow. Significantly elevated $K^{\text {trans }}$ was observed within WMLs compared with the normal-appearing WM. Intralesion BBB patency was also evaluated in terms of $\mathrm{K}_{2}$ using DSC data demonstrating increasing BBB disruption within WMLs with increased total WML volume. These findings suggest that DSC perfusion provides valuable information in assessing cSVD because MTT may be an early marker for an asymptomatic stage of the ischemic disease before $\mathrm{CBF}$ is affected and the value of $\mathrm{BBB}$ disruption as measured by $\mathrm{K}_{2}$ may shine light on the process of disease progression.

Disclosures: Linda Knutsson—RELATED: Grant: Swedish Research Council, Swedish Cancer Society*; Support for Travel to Meetings for the Study or other Purposes: Swedish Research Council, Swedish Cancer Society.* Jerry L. Prince- UNRELATED: Grants/Grants Pending: Biogen, Comments: contract on brain image processing of people with multiple sclerosis.* Peter C.M. van Zijl—UNRELATED: Grants/Grants Pending: Philips Healthcare, Comments: research, support/equipment; Payment for Lectures Including Service on Speakers Bureaus: Philips Healthcare, Comments: lectures; Patents (Planned, Pending or Issued): Philips Healthcare, Comments: patents licensed; Travel/Accommodations/Meeting Expenses Unrelated to Activities Listed: Philips Healthcare, Comments: travel to give lectures. *Money paid to the institution.

\section{REFERENCES}

1. Corriveau RA, Bosetti F, Emr M, et al. The science of vascular contributions to cognitive impairment and dementia (VCID): a framework for advancing research priorities in the cerebrovascular biology of cognitive decline. Cell Mol Neurobiol 2016;36:281-88 CrossRef Medline

2. Corriveau RA, Koroshetz WJ, Gladman JT, et al. Alzheimer's disease-related dementias summit 2016: national research priorities. Neurology 2017;89:2381-91 CrossRef Medline

3. Bath PM, Wardlaw JM. Pharmacological treatment and prevention of cerebral small vessel disease: a review of potential interventions. Int J Stroke 2015;10:469-78 CrossRef Medline

4. Wardlaw JM, Smith C, Dichgans M. Mechanisms of sporadic cerebral small vessel disease: insights from neuroimaging. Lancet Neurol 2013;12:483-97 CrossRef Medline

5. Edrissi H, Schock SC, Cadonic R, et al. Cilostazol reduces blood brain barrier dysfunction, white matter lesion formation and motor deficits following chronic cerebral hypoperfusion. Brain Res 2016;1646:494-503 CrossRef Medline
6. Wardlaw JM, Smith EE, Biessels GJ, et al. Neuroimaging standards for research into small vessel disease and its contribution to ageing and neurodegeneration. Lancet Neurol 2013;12:822-38 CrossRef Medline

7. Thrippleton MJ, Backes WH, Sourbron S, et al. Quantifying bloodbrain barrier leakage in small vessel disease: review and consensus recommendations. Alzheimers Dement 2019;15:840-58 CrossRef Medline

8. Tsai CF, Thomas B, Sudlow CL. Epidemiology of stroke and its subtypes in Chinese vs white populations: a systematic review. Neurology 2013;81:264-72 CrossRef Medline

9. Maillard P, Carmichael O, Fletcher E, et al. Coevolution of white matter hyperintensities and cognition in the elderly. Neurology 2012;79:442-48 CrossRef Medline

10. Traylor M, Lewis CM. Genetic discovery in multi-ethnic populations. Eur J Hum Genet 2016;24:1097-98 CrossRef Medline

11. Vermeer SE, Longstreth WT Jr, Koudstaal PJ. Silent brain infarcts: a systematic review. Lancet. Neurol 2007;6:611-19 CrossRef Medline

12. Li Y, Li M, Zuo L, et al. Compromised blood-brain barrier integrity is associated with total magnetic resonance imaging burden of cerebral small vessel disease. Front Neurol 2018;9:221 CrossRef Medline

13. Luciano M, Marioni RE, Valdes HM, et al. Structural brain MRI trait polygenic score prediction of cognitive abilities. Twin Res Hum Genet 2015;18:738-45 CrossRef Medline

14. Habes M, Erus G, Toledo JB, et al. White matter hyperintensities and imaging patterns of brain ageing in the general population. Brain 2016;139:1164-79 CrossRef Medline

15. Maki T, Hayakawa K, Pham LD, et al. Biphasic mechanisms of neurovascular unit injury and protection in CNS diseases. CNS Neurol Disord Drug Targets 2013;12:302-15 CrossRef Medline

16. Lo EH, Rosenberg GA. The neurovascular unit in health and disease: introduction. Stroke 2009;40:S2-3 CrossRef Medline

17. Debette S, Markus HS. The clinical importance of white matter hyperintensities on brain magnetic resonance imaging: systematic review and meta-analysis. BMJ 2010;341:c3666 CrossRef Medline

18. Jagust W. Vulnerable neural systems and the borderland of brain aging and neurodegeneration. Neuron 2013;77:219-34 CrossRef Medline

19. Fornage M, Debette S, Bis JC, et al. Genome-wide association studies of cerebral white matter lesion burden: the charge consortium. Ann Neurol 2011;69:928-39 CrossRef Medline

20. Turner ST, Jack CR, Fornage M, et al. Heritability of leukoaraiosis in hypertensive sibships. Hypertension 2004;43:483-87 CrossRef Medline

21. Verhaaren BF, Debette S, Bis JC, et al. Multiethnic genome-wide association study of cerebral white matter hyperintensities on MRI. Circ Cardiovasc Genet 2015;8:398-409 CrossRef Medline

22. Raina A, Zhao X, Grove ML, et al. Cerebral white matter hyperintensities on MRI and acceleration of epigenetic aging: the Atherosclerosis Risk in Communities Study. Clin Epigenetics 2017;9:21 CrossRef Medline

23. Silbert LC, Lahna D, Promjunyakul NO, et al. Risk factors associated with cortical thickness and white matter hyperintensities in dementia free Okinawan elderly. J Alzheimers Dis 2018;63:365-72 CrossRef Medline

24. Walker KA, Power MC, Hoogeveen RC, et al. Midlife systemic inflammation, late-life white matter integrity, and cerebral small vessel disease: the Atherosclerosis Risk in Communities Study. Stroke 2017;48:3196-3202 CrossRef Medline

25. Nam KW, Kwon HM, Jeong HY, et al. High neutrophil to lymphocyte ratio is associated with white matter hyperintensity in a healthy population. J Neurol Sci 2017;380:128-31 CrossRef Medline

26. Wong SM, Jansen JF, Zhang CE, et al. Blood-brain barrier impairment and hypoperfusion are linked in cerebral small vessel disease. Neurology 2019;92:e1669-77 CrossRef Medline

27. Markus HS, Lythgoe DJ, Ostegaard L, et al. Reduced cerebral blood flow in white matter in ischaemic leukoaraiosis demonstrated using quantitative exogenous contrast-based perfusion MRI. $J$ Neurol Neurosurg Psychiatry 2000;69:48-53 CrossRef Medline 
28. Arba F, Leigh $\mathrm{R}$, Inzitari D, et al. Blood-brain barrier leakage increases with small vessel disease in acute ischemic stroke. Neurology 2017;89:2143-50 CrossRef Medline

29. Gupta N, Simpkins AN, Hitomi E, et al. White matter hyperintensity-associated blood-brain barrier disruption and vascular risk factors. J Stroke Cerebrovasc Dis 2018;27:466-71 CrossRef Medline

30. Schumann P, Touzani O, Young AR, et al. Evaluation of the ratio of cerebral blood flow to cerebral blood volume as an index of local cerebral perfusion pressure. Brain 1998;121:1369-79 CrossRef Medline

31. Shi Y, Thrippleton MJ, Makin SD, et al. Cerebral blood flow in small vessel disease: a systematic review and meta-analysis. J Cereb Blood Flow Metab 2016;36:1653-67 CrossRef Medline

32. Zaharchuk G. Theoretical basis of hemodynamic MR imaging techniques to measure cerebral blood volume, cerebral blood flow, and permeability. AJNR Am J Neuroradiol 2007;28:1850-58 CrossRef Medline

33. Patlak CS, Blasberg RG, Fenstermacher JD. Graphical evaluation of blood-to-brain transfer constants from multiple-time uptake data. J Cereb Blood Flow Metab 1983;3:1-7 CrossRef Medline

34. Brickman AM, Zahra A, Muraskin J, et al. Reduction in cerebral blood flow in areas appearing as white matter hyperintensities on magnetic resonance imaging. Psychiatry Res 2009;172:117-20 CrossRef Medline

35. Sachdev P, Wen W, Shnier R, et al. Cerebral blood volume in T2weighted white matter hyperintensities using exogenous contrastbased perfusion MRI. J Neuropsychiatry Clin Neurosci 2004;16:83-92 CrossRef Medline

36. Knutsson L, Stahlberg F, Wirestam R. Absolute quantification of perfusion using dynamic susceptibility contrast MRI: pitfalls and possibilities. MAGMA 2010;23:1-21 CrossRef Medline

37. Nyquist PA, Bilgel M, Gottesman R, et al. Age differences in periventricular and deep white matter lesions. Neurobiol Aging 2015;36:165358 CrossRef Medline

38. Nyquist PA, Yanek LR, Bilgel M, et al. Effect of white matter lesions on manual dexterity in healthy middle-aged persons. Neurology 2015;84:1920-26 CrossRef Medline

39. Nyquist PA, Yanek LR, Kral BG, et al. White matter lesion progression and cognitive function over 5 years in a young susceptible population. Neuroepidemiology 2017;49:62-63 CrossRef Medline

40. Roy S, He Q, Sweeney E, et al. Subject-specific sparse dictionary learning for atlas-based brain MRI segmentation. IEEE J Biomed Health Inform 2015;19:1598-1609 CrossRef Medline

41. Roy S, Carass A, Prince JL, et al. Subject-specific sparse dictionary learning for atlas based brain MRI segmentation. Mach Learning Med Imaging 2014;8679:248-55 CrossRef Medline

42. O'Sullivan M, Lythgoe DJ, Pereira AC, et al. Patterns of cerebral blood flow reduction in patients with ischemic leukoaraiosis. Neurology 2002;59:321-26 CrossRef Medline

43. Becker DM, Yook RM, Moy TF, et al. Markedly high prevalence of coronary risk factors in apparently healthy African-American and White siblings of persons with premature coronary heart disease. Am J Cardiol 1998;82:1046-51 CrossRef Medline

44. Nyquist PA, Wityk R, Yanek LR, et al. Silent small-vessel cerebrovascular disease and silent myocardial ischemia in families with premature coronary disease. Neuroepidemiology 2009;33:66-67 CrossRef Medline

45. Shiee N, Bazin PL, Ozturk A, et al. A topology-preserving approach to the segmentation of brain images with multiple sclerosis lesions. Neuroimage 2010;49:1524-35 CrossRef Medline

46. Sourbron SP, Buckley DL. On the scope and interpretation of the Tofts models for DCE-MRI. Magn Reson Med 2011;66:735-45 CrossRef Medline

47. Wu O, Østergaard L, Weisskoff RM, et al. Tracer arrival timinginsensitive technique for estimating flow in MR perfusion-weighted imaging using singular value decomposition with a block-circulant deconvolution matrix. Magn Reson Med 2003;50:164-74 CrossRef Medline

48. Dewey B. ECTRIMS ONLINE LIBRARY. 2017. https://www.ectrims. eu/online-library/. Accessed March 1, 2020

49. Avants BB, Tustison NJ, Song G, et al. A reproducible evaluation of ANTS similarity metric performance in brain image registration. Neuroimage 2011;54:2033-44 CrossRef Medline

50. Roy S, Butman JA, Pham DL. Robust skull stripping using multiple MR image contrasts insensitive to pathology. Neuroimage 2017;146:132-47 CrossRef Medline

51. Huo Y, Plassard AJ, Carass A, et al. Consistent cortical reconstruction and multi-atlas brain segmentation. Neuroimage 2016;138:197-210 CrossRef Medline

52. Leigh R, Jen SS, Varma DD, et al. Arrival time correction for dynamic susceptibility contrast MR permeability imaging in stroke patients. PLoS.One 2012;7:e52656 CrossRef Medline

53. Boxerman JL, Schmainda KM, Weisskoff RM. Relative cerebral blood volume maps corrected for contrast agent extravasation significantly correlate with glioma tumor grade, whereas uncorrected maps do not. AJNR Am J Neuroradiol 2006;27:859-67 Medline

54. Oishi K, Faria A, Jiang $\mathrm{H}$, et al. Atlas-based whole brain white matter analysis using large deformation diffeomorphic metric mapping: application to normal elderly and Alzheimer's disease participants. Neuroimage 2009;46:486-99 CrossRef Medline

55. R Core Team. R: A Language and Environment for Statistical Computing. https://cran.r-project.org/doc/manuals/r-release/ fullrefman.pdf. Accessed March 1, 2020

56. Marstrand JR, Garde E, Rostrup E, et al. Cerebral perfusion and cerebrovascular reactivity are reduced in white matter hyperintensities. Stroke 2002;33:972-76 CrossRef Medline

57. Perkio J, Aronen HJ, Kangasmaki A, et al. Evaluation of four postprocessing methods for determination of cerebral blood volume and mean transit time by dynamic susceptibility contrast imaging. Magn Reson Med 2002;47:973-81 CrossRef Medline

58. Mazziotta J, Toga A, Evans A, et al. A probabilistic atlas and reference system for the human brain: International Consortium for Brain Mapping (ICBM). Philos Trans R Soc Lond B Biol Sci 2001;356:129322 CrossRef Medline

59. Bazin PL, Cuzzocreo JL, Yassa MA, et al. Volumetric neuroimage analysis extensions for the MIPAV Software Package. $J$ Neurosci Methods 2007;165:111-21 CrossRef Medline

60. Zierler KL. Equations for measuring blood flow by external monitoring of radioisotopes. Circ Res 1965;16:309-21 CrossRef Medline

61. Rempp KA, Brix G, Wenz F, et al. Quantification of regional cerebral blood flow and volume with dynamic susceptibility contrastenhanced MR imaging. Radiology 1994;193:637-41 CrossRef Medline

62. Promjunyakul NO, Lahna DL, Kaye JA, et al. Comparison of cerebral blood flow and structural penumbras in relation to white matter hyperintensities: a multi-modal magnetic resonance imaging study. J Cereb Blood Flow Metab 2016;36:1528-36 CrossRef Medline

63. van Osch MJ, Teeuwisse WM, van Walderveen MA, et al. Can arterial spin-labeling detect white matter perfusion signal? Magn Reson Med 2009;62:165-73 CrossRef Medline

64. Welker K, Boxerman J, Kalnin A, et al. ASFNR recommendations for clinical performance of MR dynamic susceptibility contrast perfusion imaging of the brain. AJNR Am J Neuroradiol 2015;36: E41-51 CrossRef Medline

65. Barnes SR, Ng TS, Montagne A, et al. Optimal acquisition and modeling parameters for accurate assessment of low $K^{\text {trans }}$ blood-brain barrier permeability using dynamic contrast-enhanced MRI. Magn Reson Med 2016;75:1967-77 CrossRef Medline 